\title{
CARMEN, LIQUID PROPULSION SYSTEMS SIMULATION PLATFORM
}

\author{
E. Cliquet ${ }^{1}$, A. Iannetti ${ }^{1}$, and J. Masse ${ }^{2}$ \\ ${ }^{1}$ Centre National d'Etude Spatiales (CNES) \\ Evry, France \\ ${ }^{2}$ APPEDGE \\ Nanterre, France
}

CNES Launchers Directorate (DLA) has to maintain a high level of expertise to achieve its missions regarding the exploitation of the Ariane 5 family launchers and to prepare the future of launchers. For this purpose, developing and using numerical simulation tools is of key importance. CARMEN software is CNES reference tool for simulating propulsion systems from their design to their exploitation. CARMEN regroups several CNES software packages. Among them are: CARDIM, for engine thermodynamics and geometric design, and CARSTAT/CARINS, for analyzing functional behavior of propulsion systems in stationary (CARSTAT) or transient (CARINS) modes. This paper extensively presents CARMEN software development status and capabilities.

\section{INTRODUCTION}

CNES Launchers Directorate has to maintain a high level of expertise to achieve its missions regarding the exploitation of the Ariane 5 family of launchers and to prepare the future of launchers. For this purpose, developing and using numerical simulation tools is of key importance. Furthermore, having a software that allows capitalizing the know-how and technical skills in different disciplines encountered in propulsion is a strong asset for CNES.

CARMEN is the DLA software platform that regroups all tools necessary for liquid propellant system design and analysis. As can be seen in Fig. 1, CARMEN is divided in two modules: CARDIM and CARFONC. CARDIM allows the calculation of engine thermodynamic cycle (CARMOT) and the preliminary geometric design of subsystems (CARSYS). CARFONC allows predictions and analysis of steady-state operation (CARSTAT) and transients (CARINS).

This is an Open Access article distributed under the terms of the Creative Commons Attribution-Noncommercial License 3.0, which permits unrestricted use, distribution, and reproduction in any noncommercial medium, provided the original work is properly cited. 


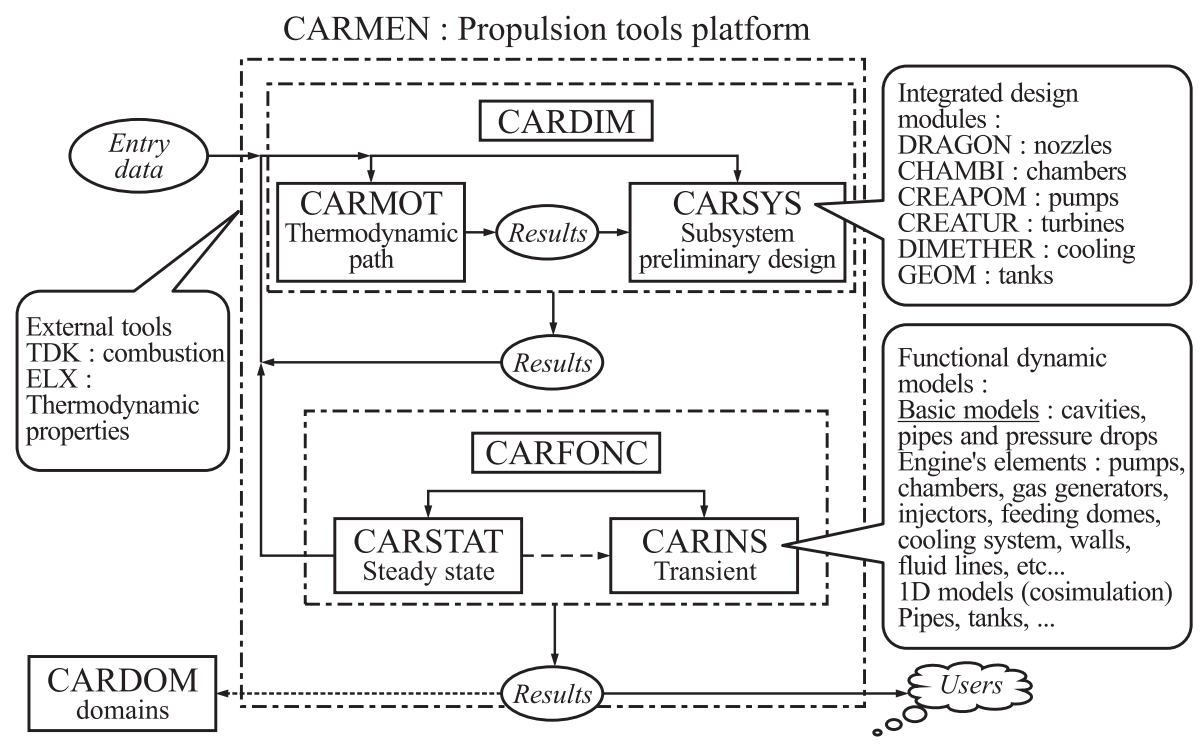

Figure 1 CARMEN software platform

These modules are designed to be capable of communicating easily with each other: they have a common graphic user interface (GUI) written in JAVA and can exchange and use relevant results files.

CARMOT, CARSTAT, and CARINS are based on symbolic formulas manipulation with a computer algebra system named MAXIMA which is the open source (http://maxima.sourceforge.net). This method allows splitting the simulation in three steps as shown in Fig. 2:

(1) modeling through the GUI which consists in choosing components and associated models in a library and connecting them;

(2) automatic generation of a dedicated and optimized executable simulator linked with a solver: this is achieved through the sorting out of the equations by MAXIMA, creation and compilation of a FORTRAN code, and linking with the solver which differs between CARDIM and CARFONC; and

(3) simulation and exploitation of the results through the GUI.

After achieving these steps, the user has access to several features depending on the module such as parametric studies, sensitivity analyses, etc.

The distinguishing feature of CARMEN is the possibility given to the user to implement his or her own physical models directly through the GUI. The user does not need to know how to code, since the equations can be written literally 


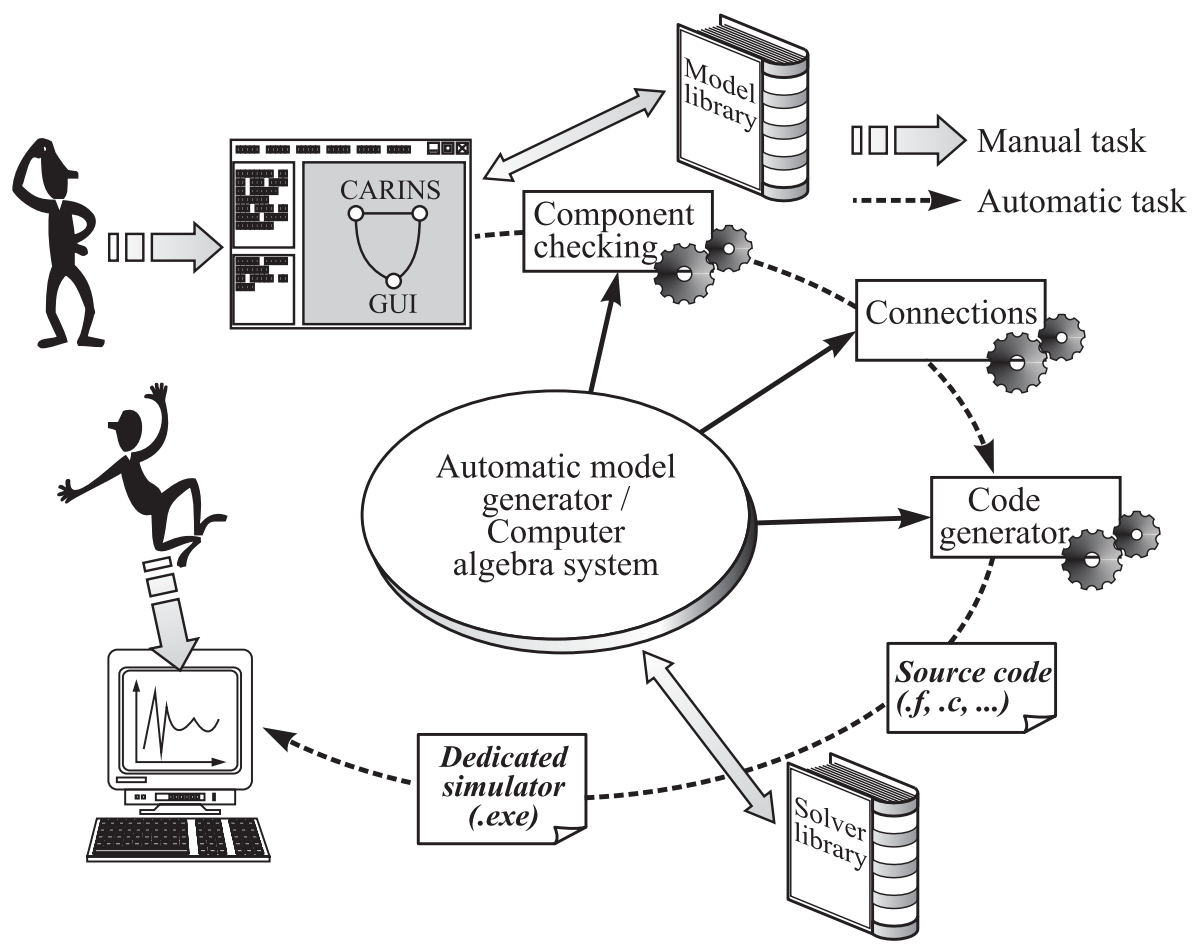

Figure 2 Operating mode common to CARMOT, CARSTAT, and CARINS

through a dedicated tool of the interface and associated with an engine element in a customized library. In case of need, the user can write his or her own models using several languages such as FORTRAN, C, or even MAXIMA.

Moreover, CARMEN is also capable of communicating with other codes like CARMECA [1], an in-house developed tool for preliminary mechanical analyses.

CARMEN is currently under development within the frame of the MINOS project [2] and its development was started in 2009 with the second version of CARINS. The development of this new version of CARINS has been completed and all the features of CARINS are presented in the paper along with the comparison between simulator results and hardware tests. Then CARDIM was developed between 2007 and 2009, in order to replace CARAMEL [3], a similar tool developed in the 1990s. The new born in the family is CARSTAT and its development is just beginning. CARSTAT is based on CARINS experience and its basic principles will also be discussed in this paper in detail. 


\section{CARFONC}

\subsection{CARINS}

\subsubsection{General Outline}

From 2002 to 2005, CNES DLA, along with ONERA, APPEDGE, CAPGEMINI and several university laboratories developed the open software CARINS $[4,5]$. CARINS is a versatile and flexible tool for reproducing time evolution of physical parameters which characterize the behavior of space propulsion systems or subsystems, especially during transient phases. It can be used in the advanced design phases of a space propulsion subsystem as well as for ground tests or flights analysis.

From 2006 to 2009, a new version of CARINS has been developed, including a new GUI, new models, and new features.

Thanks to its architecture based on MAXIMA as described in Introduction, CARINS is capable of simulating all systems that can be described by ordinary differential or algebraic equations. Moreover, CARINS also has the capability of using cosimulation for problems described by more complex types of governing equations.

CARINS relies on its library of high level physical models, which are developed by ONERA, LML (Laboratoire de Mécanique de Lille) [6, 7], LFCT (Laboratoire des fluides complexes et thermique), and LEMTA (Laboratoire d'Énergétique et de Mécanique Théorique et Appliquée) [8]. Among others, this library contains the following models :

- transient models of pumps and turbines;

- combustion chambers taking into account chemical kinetics and droplet evaporation (for nonideal and ideal perfect gases);

- models of cavities, orifices, and one-dimension (1D) cosimulated nonadiabatic pipes; available for ideal and nonideal perfect gases, real gases (e. g., Benedict-Webb-Rubin equation of state), and two-phase flows; and

- 1D model of axisymmetric tank using cosimulation.

CARINS also offers several tools based on SCILAB to perform further studies with the help of the GUI. Parametric studies and sensitivity analysis are available, along with an optimization module to calibrate simulations against test results.

\subsubsection{Some Examples of Using CARINS}

This subsection describes some transient simulators which are available or under development at CNES Directorate of Launchers. 


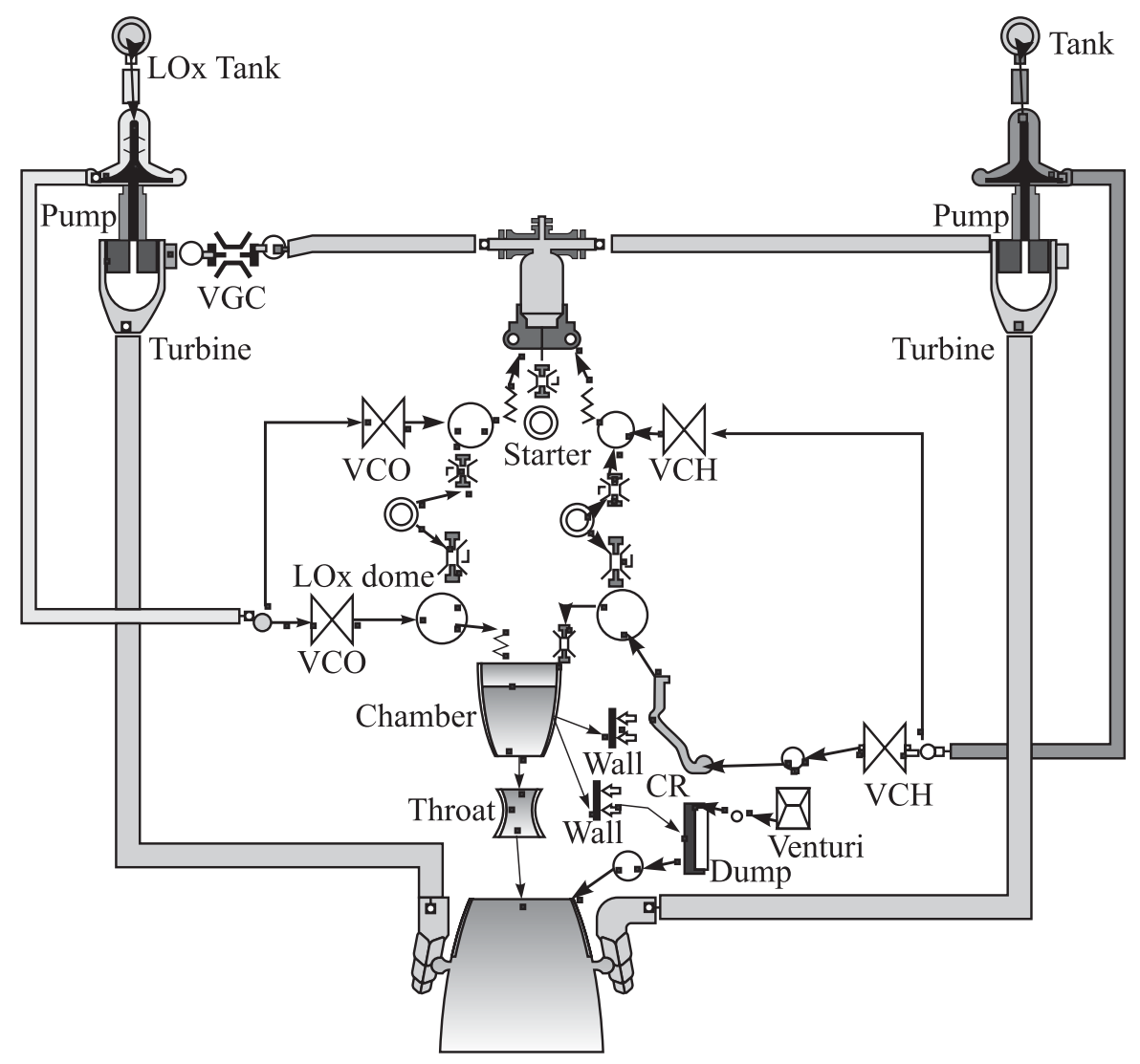

Figure 3 Vulcain 2 synoptic in CARINS V2

\section{Vulcain 2 Engine Start-Up}

The simulator of Vulcain 2, Ariane 5 main cryogenic stage engine, in CARINS has been developed using the models from CARINS main library and some specific models purposefully created for it through the GUI of CARINS, before the newest models developed for the second version of CARINS were available. Figure 3 shows how the engine looks in the newest version of CARINS and Fig. 4 shows the computed oxygen turbopump rotational speed compared to ground test results. The computation time for this simulation is $4 \mathrm{~min}$.

\section{EPC Stage Simulator}

Two versions of Ariane 5 cryogenic main stage simulator have been developed with the first version of CARINS, one for the Ariane 5 Generic configuration and 


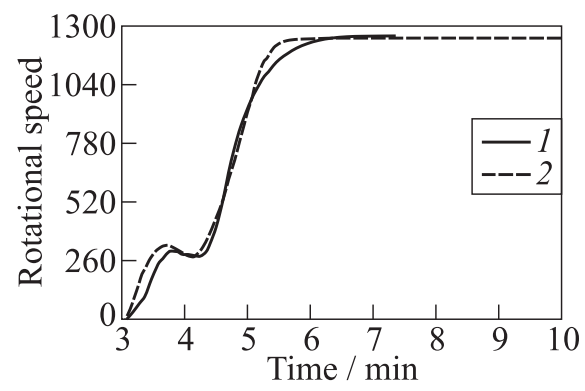

Figure 4 Comparison between simulated (1) and measured (2) values of Vulcain 2 oxygen pump rotational speed

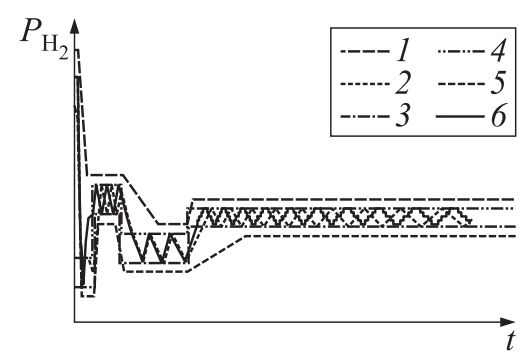

(a)

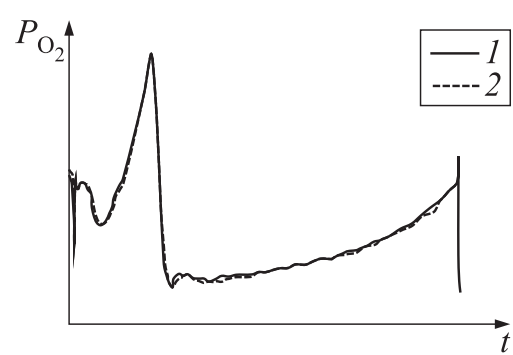

(b)

Figure 5 Etage principle cryotechnique (EPC) hydrogen tank pressure $(a)$ and oxygen pump pressure (b): 1 - flight; 2 - simulation; 3 and 4 - lower and higher thresholds; and 5 and 6 - lower and higher envelopes, respectively

the other for Ariane 5 ECA configuration. Both simulators have been compared to flight measurements and give very good results except for the temperature of high-pressure Helium which was modeled as a perfect gas since the real gas model was not available at that time. Figure 5 shows some of the results compared to Ariane 5 flight measurements. The computation time is less than $15 \mathrm{~min}$ for several hundred seconds of real time. This model is to be assembled with the Vulcain 2 engine simulator.

\section{EPC Stage Simulator}

For the delivery of the Automated Transfer Vehicle, Ariane 5 hydrazine attitude control system (SCA) had to be modified and requalified for long-duration missions. The corresponding version of the attitude control system is called SCA-VUS for Versatile Upper Stage. Thanks to CARINS, cross-checks of ground tests and flight predictions became possible. Figure 6 compares CARINS and Thermal-Mathematical Model (TMM) flight predictions. 
Among other CARINS simulators, the most advanced ones are VULCAIN, VINCI, and HM7B engines, VEGA launcher's AVUM stage, and KVD-1 LOx-Methane engine. VULCAIN and HM7B engine simulators show good results for start-up transients. VINCI engine simulator is very robust and has less than $3 \%$ error for 8 different steady operation points. AVUM stage simulator includes the pressurization system, tanks, manifolds, and the MEA engine and has served to determine the domain of pressure and temperature at engine

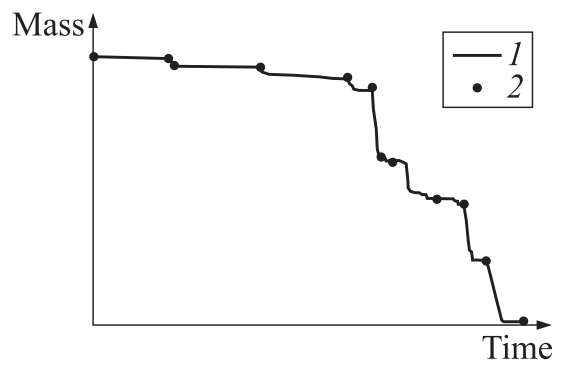

Figure 6 SCAVUS liquid mass of hydrazine in tanks prediction: 1 CARINS; and 2 - TMM inlet during "hot" and "cold" missions, taking into account external thermal fluxes (convection and radiation). KVD-1 simulator [9] has served to reproduce ground tests made in cooperation with Russia [10].

\subsubsection{Concluding Remarks}

CARINS is now widely used by CNES DLA propulsion engineers. Its easiness of use and reliability allow them to create optimized and dedicated simulators on demand at subsystem or system level, with good reactivity.

Future developments of CARINS are also planned, including coupling CARINS with three-dimensional (3D) codes or enlarged real-fluid thermodynamic tables.

\subsection{CARSTAT}

\subsubsection{General Outline}

CARSTAT will be the tool dedicated to steady-state operation analysis and prediction. It will allow simulating steady-state operations and engine tuning as well as limit, extreme, and qualification domains. Thus, CARSTAT will be used for advanced design phases of new liquid-propellant propulsion systems (calculation of operation points of engine designed by CARDIM) as well as for the exploitation phase of existing propulsion systems (operation points prediction, domain analysis).

CARSTAT will be able to dialog with CARDIM and CARINS as well as with codes external to CARMEN, like the domain-analysis code CARDOM, following the flux diagram shown in Fig. 7. 


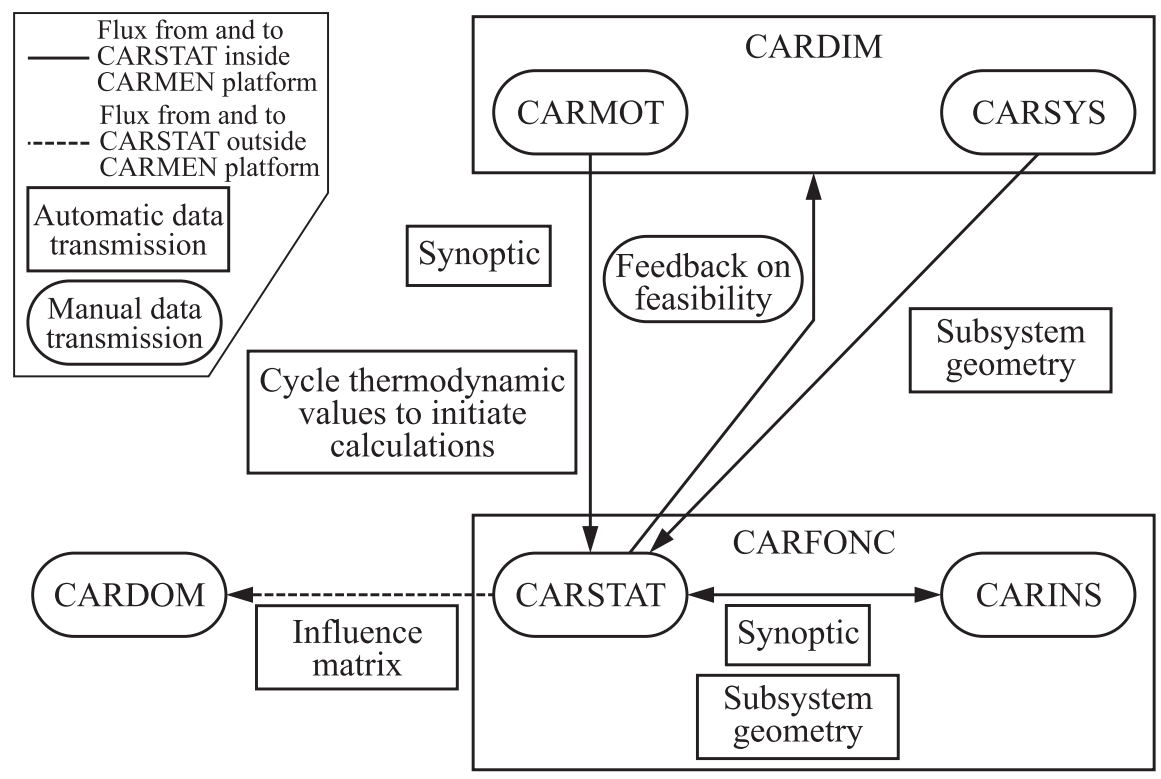

Figure 7 Flux diagram of information and data exchanges between CARSTAT and the other codes

CARDOM is an in-house software that allows the calculation of functional domains of an engine starting from the influence matrix or from a Monte Carlo calculation of engine variables.

Like CARINS, CARSTAT will be capable of performing parametric studies, sensitivity analyses, and parametric identification.

\subsubsection{CARSTAT Strategy}

In most cases, the software aimed at simulating steady-state operation is based on iterative solvers using Newton methods. The experience acquired with CARINS shows that CARINS solvers (LSODA - Livermore Solver for Ordinary Differential Equations, LSODES) are very efficient.

The time taken by those solvers to reach a stabilized regime essentially depends on the stiffness of the system of equations to be solved. The aim of CARSTAT is to reuse CARINS physical models and solvers and to reduce their stiffness in order to reach the stabilized regime solution as soon as possible. This will be done by modifying physical models to suppress fast phenomena while keeping exact solution for steady state. Thus, the transients observed with these models will have no more physical significance but will be a mean to rapidly reach the steady-state solution. 
Apart from the very short simulation time expected with this method, the use of this strategy will be transparent for the user which will only be given the steady-state solution. However, if needed, the user will also be able to observe the "mathematical transient" when working with personal physical models.

\subsubsection{Concluding Remarks}

CARSTAT tool will be based on an inventive methodology, benefiting from CARINS experience, which consists in transforming the physical transients of existing models in a mathematical transient. This method should be able to provide very fast and reliable simulations.

\section{CARDIM}

\subsection{General Outline}

Following the success of CARINS development, CNES with its partners APPEDGE, and CAPGEMINI decided to develop a new software dedicated to the preliminary design phase of engines.

Developed between 2007 and 2009, CARDIM employs the same informatics as CARINS. It is composed of two main modules: CARMOT for the calculation of engine thermodynamic cycle and CARSYS for the preliminary geometric design of subsystems, thus providing a practical tool for concurrent engineering design approaches.

CARDIM offers a complete set of thermodynamic models for engine cycle calculations (CARMOT) and a library of advanced design modules for subsystem design (CARSYS). The CARSYS modules are developed independently by each specialist and customized for use in CARDIM. They are:

- CREAPOM and CREATUR for turbopumps design;

- CHAMBI for thrust chamber and injectors;

- DRAGON for nozzles; and

- DIMETHER for regenerative circuits.

As in CARINS, the user can easily create and manage his own models directly via the GUI thanks to the personal libraries.

Back into the CARMEN design logic, once the engine preliminary design is completed in CARDIM, the geometrical data of the corresponding subsystems are transferred to CARINS (or CARSTAT) for simulating the transient behavior as well as to CARMECA [1] for preliminary mechanical analyses. 


\subsection{CARDIM: Test Case}

Shown in this subsection are the results for the CARDIM design of the Vulcain engine, previous main engine of Ariane 5. Starting on from the overall system specification, CARDIM provides the first preliminary design of the cycle and the subsystems (Table 1 and 2).

The thermodynamic scheme of an engine is built in the CARMOT interface. A set of initial guess data for the unknown parameters is imposed. The VULCAIN
Table 1 Input data for the Vulcain engine

\begin{tabular}{lc}
\hline \multicolumn{1}{c}{ Parameter } & Meaning \\
\hline Vacuum thrust & $1025 \mathrm{kN}$ \\
$P_{c}$ & $100 \mathrm{bar}$ \\
$P_{c}$ (gas generator) & $77.4 \mathrm{bar}$ \\
$T_{c \text { max }}$ (gas generator) & $870 \mathrm{~K}$ \\
$R_{M}$ (chamber) & 5.9 \\
$R_{M}$ (gas generator) & 0.9 \\
\hline
\end{tabular}
thermodynamic model presents 155 variables and a total of 58 equations. The simulator provides the system solution in about a minute.

The subsystem design part is done with CARSYS. Table 3 shows the percent differences between the simulated and the real geometry of the Vulcain engine whereas Tables 4 and 5 are related to oxygen turbine and to the divergent part of the nozzle. The results give the estimates of the real geometry with a maximum difference of $10 \%$ : this depends on the answers and values assigned by default to the module. This approach is meant to provide the first approximation for the

Table 2 CARMOT results and reference values for the Vulcain engine

\begin{tabular}{ccccc}
\hline Case & $\begin{array}{c}\text { Vacuum thrust, } \\
\mathrm{kN}\end{array}$ & $\begin{array}{c}\text { Vacuum } I_{\mathrm{sp}}, \\
\mathrm{s}\end{array}$ & $\begin{array}{c}\text { Hydrogen } \\
\text { turbopump } \\
W, \mathrm{MW}\end{array}$ & $\begin{array}{c}\text { Oxygen } \\
\text { turbopump } \\
W, \mathrm{MW}\end{array}$ \\
\hline Reference & 1025.0 & 433.3 & 10.3 & 2.8 \\
CARDIM data & 1024.3 & 433.53 & 10.29 & 2.8 \\
\hline$\Delta$ & $<1 \%$ & $<1 \%$ & $<1 \%$ & $<1 \%$ \\
\hline
\end{tabular}

Table 3 Comparison of the geometrical results for the combustion chamber

\begin{tabular}{lc}
\hline Combustion chamber & Results vs. real data, \% \\
\hline \multicolumn{2}{c}{ Chamber body } \\
\hline Throat diameter & -6 \\
Chamber diameter & -2 \\
Total chamber length & +1 \\
Convergent angle & -3 \\
\hline \multicolumn{1}{c}{ Injectors } \\
\hline Number of elements & -12 \\
Mass flow per element & +11 \\
\hline
\end{tabular}


Table 4 Comparison of the results for the Oxygen turbine

\begin{tabular}{lccc}
\hline Case & $\begin{array}{c}\text { Number } \\
\text { of centrifugal stages } \\
\text { for TPO pump }\end{array}$ & $\begin{array}{c}\text { Number } \\
\text { of centrifugal stages } \\
\text { for TPO turbine }\end{array}$ & $\begin{array}{c}\text { Rotational } \\
\text { speed TPO, } \\
\text { rpm }\end{array}$ \\
\hline Reference & 1 & 1 & 12530 \\
CARDIM & 1 & 1 & 12530 \\
\hline Results vs. real data, $\%$ & 0 & 0 & 0 \\
\hline
\end{tabular}

Table 5 Comparison of results for the divergent part

\begin{tabular}{lc}
\hline Divergent part & Results vs. real data, $\%$ \\
\hline Divergent length & +6 \\
Exit diameter & +5 \\
\hline
\end{tabular}

design and it is always possible to refine the results using the single module in its full advanced version without the simplifications integrated in CARDIM.

The nozzle detailed profile is sketched via the DRAGON module. Figure 8 shows the results obtained.

The preliminary design obtained with the initial values can be further improved via the refinement of the parameters of each particular module of

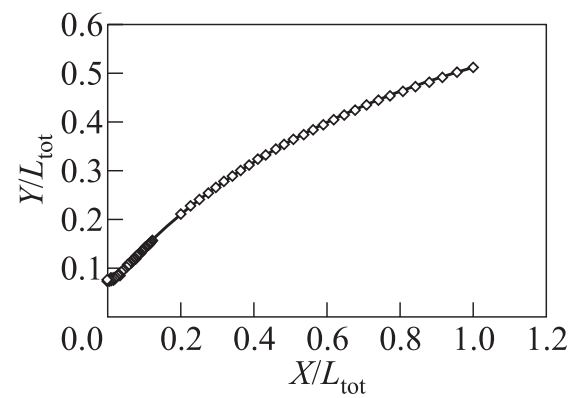

Figure 8 Nozzle contour results CARSYS, but it still provides representative calculations of the engine functional performances and constitutes a basis for comparison and further analysis.

\section{CONCLUDING REMARKS}

In this paper, CARMEN, the software platform for liquid propulsion systems simulation currently used at CNES Directorate of Launchers has been illustrated, showing its present capabilities and future development concepts. CARMEN is composed of two main modules, each of them divided into two submodules dedicated to a specialized part of the propulsion system design:

(1) CARDIM module, composed of CARMOT for the calculation of the engine thermodynamic cycle and by CARSYS for the preliminary geometric design of propulsion subsystems and elements; and 
(2) CARFONC module, composed of CARINS suited for transient analyses and CARSTAT for the analyses of engine steady states.

Once the engine preliminary design is completed via the use of CARDIM module, the geometrical data of the subsystems can be used in CARFONC to obtain the steady-state or transient behavior, as well as in CARMECA for the preliminary mechanical analysis.

CARMEN submodules are linked together and each output file can be exploited in various forms. The entire cycle of engine analysis is implemented in a common and unique software environment.

\section{REFERENCES}

1. Ferlin, M., and D. Tchou-Kien. 2009. CARMECA, Propulsion tool for a rapid rocket engine sizing solution. 3rd EUCASS Conference. Versailles, France.

2. Illig, M., P. Mourey, and M. Malnar. 2008. MINOS, a project for a launcher system simulation. 2nd Symposium (International) on Propulsion for Space Transportation, Space Propulsion. Heraklion, Greece.

3. Espinosa-Ramos, A. 2002. CARAMEL: The CNES computation software for designing liquid rocket engines. 4th Conference (International) on Launcher Technology "Space Launcher Liquid Propulsion." Liege, Belgium.

4. Leudiere, V., G. Albano, G. Ordonneau, J. Masse, and B. Legrand. 2004. CARINS: A versatile and flexible tool for engine transient prediction - development status. 24th Symposium (International) on Technologies for Space. Tokyo, Japan.

5. Ordonneau, G., G. Albano, V. Leudiere, and J. Masse. 2005. CARINS: A new versatile and flexible tool for engine transient prediction - development status. 6th Symposium (International) on Launcher Technologies. Munich, Germany.

6. Corvisier, P., M. Lazard, M. Feidt, and G. Albano. 2003. Non ideal gas flow simulation: A new formulation of conservation equations. Launcher Technology European J. Mechan. Environ. Eng. 48(2):67-72. ISSN 1371-6980.

7. Dazin, A., G. Caignaert, and G. Bois. 2006. Experimental and theoretical analysis of a centrifugal pump during fast starting period. 11th Symposium (International) on Transport Phenomena and Dynamics of Rotating Machinery "ISROMAC 11." Hawaii, USA.

8. Jacquot, C., M. Feidt, P. Corvisier, and G. Albano. 2003. Transient convective heat transfer in single and two phase flow: Application to cryogenic heat exchangers. ICHMT Symposium (International) on Transient Convective Heat and Mass Transfer in Single and Two-Phase Flows. Izmir, Turkey.

9. Leudiere, V., P. Supie, A. Beaurain, and M. Villa. 2007. KVD-1 engine in LOx/ $\mathrm{CH}_{4}$. 43rd AIAA/ASME/SAE/ASEE Joint Propulsion Conference. Cincinnati, USA.

10. Bonhomme, C., and M. Theron. 2006. French/Russian activities on LOx/ $/ \mathrm{LCH}_{4}$ area. 57th Astronautical Congress (International). Valence, Spain. 